\title{
Astragaloside IV Alleviates Tacrolimus-Induced Chronic Nephrotoxicity via p62-Keap1-Nrf2 Pathway
}

\author{
Ping Gao ${ }^{1 \dagger}$, Xiaoyi $\mathrm{Du}^{2,3+}$, Lili Liu ${ }^{4}$, Hua Xu ${ }^{1}$, Maochang Liu ${ }^{1}$, Xinlei Guan ${ }^{5 *}$ and \\ Chengliang Zhang ${ }^{6 *}$
}

${ }^{1}$ Department of Clinical Pharmacy, Wuhan Children's Hospital, Tongji Medical College, Huazhong University of Science and Technology, Wuhan, China, ${ }^{2}$ Department of Pediatrics, Union Hospital, Tongji Medical College, Huazhong University of Science and Technology, Wuhan, China, ${ }^{3}$ Department of Pediatrics, Maternal and Child Hospital of Hubei Province, Tongji Medical College, Huazhong University of Science and Technology, Wuhan, China, ${ }^{4}$ Department of Pathology, Wuhan Children's Hospital, Tongji Medical College, Huazhong University of Science and Technology, Wuhan, China, ${ }^{5}$ Department of Pharmacy, Wuhan Fourth Hospital, Puai Hospital, Tongji Medical College, Huazhong University of Science and Technology, Wuhan, China, ${ }^{6}$ Department of Pharmacy, Tongji Hospital, Tongji Medical College, Huazhong University of Science and Technology, Wuhan, China

Shenzhen Traditional Chinese Medicine Hospital, The Fourth Clinical Medical College of Guangzhou University of Chinese Medicine, China

Reviewed by: Suat Ekin,

Yüzüncü YIl University, Turkey

Guoliang Dai,

Affiliated Hospital of Nanjing University of Chinese Medicine, China

Parveen Kumar,

Pt. Jawahar Lal Nehru Government Medical College And Hospital, India

*Correspondence: Xinlei Guan xinleiguan@163.com

Chengliang Zhang clzhang@tjh.tjmu.edu.cn

${ }^{t}$ These authors share first authorship

Specialty section:

This article was submitted to Renal Pharmacology,

a section of the journal

Frontiers in Pharmacology

Received: 25 September 2020

Accepted: 10 December 2020

Published: 18 January 2021

Citation:

Gao P, Du X, Liu L, Xu H, Liu M, GuanX and Zhang $C$ (2021) Astragaloside IV Alleviates Tacrolimus-Induced Chronic Nephrotoxicity via p62-Keap1Nif2 Pathway.

Front. Pharmacol. 11:610102. doi: 10.3389/fphar.2020.610102
Tacrolimus-induced chronic nephrotoxicity (TIN) hinders its long-term use in patients. However, there are no drugs available in the clinic to relieve it at present. Astragaloside IV (AS-IV) is a saponin extract of the Astragalus which is widely used in the treatment of kidney disease. This study aimed to investigate the effect of AS-IV on TIN and its underlying mechanism. Herein, C57BL/6 mice were treated with tacrolimus and/or AS-IV for 4 weeks, and then the renal function, fibrosis, oxidative stress and p62-Keap1-Nrf2 pathway were evaluated to ascertain the contribution of AS-IV and p62-Keap1-Nrf2 pathway to TIN. Our results demonstrated that AS-IV significantly improved renal function and alleviated tubulointerstitial fibrosis compared with the model group. The expression of fibrosisrelated proteins, including TGF- $\beta_{1}$, Collagen I and $\alpha$-SMA, were also decreased by AS-IV. Furthermore, AS-IV relieved the inhibition of tacrolimus on antioxidant enzymes. The data in HK-2 cells also proved that AS-IV reduced tacrolimus-induced cell death and oxidative stress. Mechanistically, AS-IV markedly promoted the nuclear translocation of Nrf2 and the renal protective effects of AS-IV were abolished by Nrf2 inhibitor. Further researches showed that phosphorylated p62 was significantly increased after AS-IV pretreatment. Moreover, AS-IV failed to increase nuclear translocation of Nrf2 and subsequent antioxidative stress in HK-2 cells transfected with p62 siRNA. Collectively, these findings indicate that AS-IV relieve TIN by enhancing p62 phosphorylation, thereby increasing Nrf2 nuclear translocation, and then alleviating ROS accumulation and renal fibrosis.

Keywords: astragaloside IV, tacrolimus, chronic nephrotoxicity, p62-Keap1-Nrf2 pathway, oxidative stress

\section{INTRODUCTION}

Tacrolimus is an immunosuppressant drug which is extensively used in organ transplantation and other autoimmune diseases (Hart et al., 2019; Gao et al., 2020). However, tacrolimus therapy is often associated with irreversible nephrotoxicity that eventually progresses to chronic kidney disease (CKD) (Ojo et al., 2003). It is estimated that $16.5 \%$ of patients develop 
tacrolimus-induced chronic nephrotoxicity (TIN) (Chapman, 2011). Despite years of study, there are no drugs available in the clinic to relieve TIN. The most commonly used risk mitigation strategies like monitoring levels to guide dosing and tacrolimus dose limitation were usually associated with increased rejection risk (Sawinski et al., 2016). Hence, it is necessary to discover a drug that can alleviate TIN and is safe for long-term use.

Oxidative stress plays a crucial role in TIN. Tacrolimus can induce renal vasoconstriction, and hence lead to renal hypoperfusion and hypoxia-reoxygenation injury and subsequently to the formation of reactive oxygen species (ROS) or free radicals. Meanwhile, tacrolimus owns a direct effect in the generation of ROS, although the underlying mechanism remains to be elucidated (Naesens et al., 2009). Consequently, the excessive ROS causes irreversible damage of the renal architecture, which is mainly manifested as striped interstitial fibrosis (Lusco et al., 2017). Therefore, anti-oxidative stress therapy may be a potential candidate for TIN (Lim et al., 2017; Luo et al., 2019).

Astragalus membranaceus, also known as Huangqi, is a traditional Chinese medicine widely used in the treatment of kidney disease (Zhang et al., 2019). A large number of clinical observations have demonstrated that Astragalus is safe for long-term use (Lin et al., 2019). Astragaloside IV (AS-IV), a saponin extract of the Astragalus root, is one of the main active ingredients of Astragalus. In recent years, some evidence indicated that AS-IV had significant renal protective effect and can attenuate renal fibrosis (Guo et al., 2016; Wang et al., 2020). In addition, AS-IV has been reported to attenuate cisplatin-induced acute kidney injury (Yan et al., 2017; Qu et al., 2020), but its effect on drug-associated chronic nephropathy has rarely been studied.

Anti-oxidative stress is one of the main mechanisms for the renoprotection activity of AS-IV (Zhang et al., 2020). Nuclear factor erythroid-related factor 2 (Nrf2) is the primary defense mechanism against oxidative stress, driving transcription of $>300$ antioxidant response element-regulated genes (Zhang and Chapman, 2020). Previous researches have suggested that ASIV could protect renal cells from oxidative stress-induced injury by activating Nrf2 (Chen et al., 2018; Wang et al., 2019a). However, the mechanism by which AS-IV activates Nrf2 remains to be elucidated. Nrf2 is negatively regulated by Kelch-like ECH-associated protein 1 (Keap1) under normal conditions. When the cell is insulted by oxidative stress, Nrf2 dissociates from Keap1, translocates into the nucleus and promotes the transcription of antioxidant genes. Notably, SQSTM1/p62 (referred to as p62 hereafter) has been recently identified as a pivotal regulator of the Keap1-Nrf2 pathway through Keap1 binding (Deng et al., 2020). Whether AS-IV activates Nrf2 through the p62-Keap1-Nrf2 pathway remains to be studied.

Therefore, the objective of our study was to evaluate whether AS-IV can alleviate TIN, and then to investigate the role of p62-Keap1-Nrf2 pathway in the renoprotective activity of AS-IV.

\section{METHODS}

\section{In Vivo Experiments}

Animal studies were performed in accordance with ethical guidelines for animal studies. All protocols received approval from the Puai Hospital Animal Care and Use Committee (KY2016-006-01). Eight-week old C57BL/6 male mice (SPF Biotechnology Co.,Ltd., Beijing) were housed with a 12-h light/dark cycle with water ad libitum. All mice were placed on a low sodium diet $(0.01 \%$ sodium diet) for 7 days prior to treatment and continued on this diet throughout the treatment period.

After acclimation for one week, weight-matched mice were randomized into five groups $(n=8)$ : control, model, AS-IV low-, middle- and high-dose groups. Mice in control group were subcutaneously given $10 \mathrm{ml} / \mathrm{kg} / \mathrm{d}$ vehicle (olive oil; Sinopharm Chemical Reagent Co., Ltd., China). Mice in model group were subcutaneously injected with $1.5 \mathrm{mg} / \mathrm{kg} / \mathrm{d}$ tacrolimus (Aladdin Reagent Co., Ltd., Shanghai, China) for 4 weeks. In addition to tacrolimus, mice in AS-IV groups were simultaneously given ASIV at 10,20 or $40 \mathrm{mg} / \mathrm{kg} / \mathrm{d}$ (KT201901, purity $\geq 98 \%$, Jintaihe Pharmaceutical Chemical Technology Co. Ltd., Chengdu, China) by oral gavage for 4 weeks. The dosage of AS-IV was determined according to previous researches (Zhou et al., 2017; Cao et al., 2019) and pre-experimental results. At the end of the treatment period, the mice were weighed and then placed in metabolic cages for the measurement of urine volume over $24 \mathrm{~h}$. On the following day, the mice were sacrificed, and blood and the kidney were obtained for further use.

\section{Kidney Histology}

After rinsing with PBS solution, kidney tissue samples were fixed in $10 \%$ buffered formaldehyde and embedded in paraffin. Renal histological changes were assessed using hematoxylin-eosin and Masson trichrome staining to analyze renal pathology and fibrosis. Tubulointerstitial fibrosis was defined as a matrix-rich expansion of the interstitium with tubular dilatation, tubular atrophy, tubular cast formation, sloughing of tubular epithelial cells, or thickening of the tubular basement membrane in Masson trichrome-stained tissue sections (Lim et al., 2015). The extent of fibrosis was estimated in minimum of 10 fields per section by counting the percentage of injured area per field using the Image Pro plus software (Media Cybernetics, SilverSpring, United States). Histopathological analysis was performed in randomly selected cortical fields of sections by a pathologist blinded to the identity of the treatment groups.

\section{Biochemical Assay}

The levels of serum creatinine (Scr) and blood urea nitrogen (BUN) were measured with assay kits (Jiancheng bioengineering institute, Nanjing, China). The assay for malondialdehyde (MDA) content was performed according to the protocols of the MDA kit (A003-1-2, Jiancheng bioengineering institute, Nanjing, China). And the enzyme activity of superoxidase dismutase (SOD), catalase (CAT) and glutathione peroxidase (GSH-Px) were examined by the test kits (A001-3-2, 
A007-2-1, A005-1-2, Jiancheng bioengineering institute, Nanjing, China). The detailed experimental protocols were provided in supplementary materials.

\section{Western Blotting}

The tissue was pelleted by brief centrifugation, resuspended in ice-cold lysis buffer containing $50 \mathrm{mM}$ Tris- $\mathrm{HCl}, 100 \mathrm{mM} \mathrm{NaCl}$, 1\% Nonidet P-40, $10 \mathrm{mM}$ EDTA, $20 \mathrm{mM} \mathrm{NaF}, 1 \mathrm{mM}$ PMSF, $3 \mathrm{mM} \mathrm{Na} 3 \mathrm{VO} 4$ and protease inhibitor mixture, homogenized thoroughly, and centrifuged $\left(12,000 \mathrm{~g}\right.$ for $15 \mathrm{~min}$ at $\left.4^{\circ} \mathrm{C}\right)$. The supernatant fraction was aliquoted and stored at $-80{ }^{\circ} \mathrm{C}$ before using for Western blot. Western blot analysis procedures were processed according to our previous protocols (Zhang et al., 2018). Images were captured with Micro Chemi (DNR Bioimaging systems, Israel) and NIH ImageJ software was used to quantify the detected bands. $\beta$-actin was used as loading control and all assays were performed at least three times.

\section{Reverse Transcriptase-Polymerase Chain Reaction (RT-PCR)}

Total RNA in kidney tissues of mice was isolated with TransZol reagent (TransGen, Beijing, China). Total RNA $(1 \mu \mathrm{g})$ in each sample was reversely transcribed into cDNA using a PrimeScript RT Master Mix (Takara Shuzo, Shiga, Japan) according to the manufacturer's instructions. One $\mu \mathrm{l}$ of the resulting cDNA was used in polymerase chain reaction (PCR). The following primers were employed: Nrf2 primers (forward: $5^{\prime}$-CTCGCTGGAAAA AGAAGTG-3'; reverse: $5^{\prime}$-CCGTCCAGGAGTTCAGAGG-3'), heme oxygenase-1 (HO-1) primers (forward: CAGGGTGAC AGAAGAGGCTAAGAC, reverse: TTGTGTTCCTCTGTC AGCATCAC), NAD(P)H:quinone oxidoreductase 1 (NQO1) primers (forward: 5'-GGAAGCTGCAGACCTGGTGA-3'; reverse: $\quad 5^{\prime}$-CCTTTCAGAATGGCTGGCA-3 $\left.{ }^{\prime}\right)$, glutamatecysteine ligase catalytic subunit (GCLC) primers (forward: $5^{\prime}$ CACTGCCAGAACACAGACCC- ${ }^{\prime}$; reverse: $5^{\prime}$-ATGGTCTGG CTGAGAAGCC T-3'), Keap1 primers (forward:5' AAGGACCTTGTGGAAGACCA- $3^{\prime}$; reverse: $5^{\prime}$-CCCTGTCCA CTGGAATTGAT- $3^{\prime}$ ), p62 primers (forward: $5^{\prime}$-ATGGGACGC TGACTCACTGC- $3^{\prime}$; reverse: $5^{\prime}$-GAAGCACAGAAGAGGGAG TCT $-3^{\prime}$ ) and GAPDH primers (forward: $5^{\prime}$-CAAGGTCATCCA TGACAACTTTG-3' ${ }^{\prime}$; reverse: $5^{\prime}$ - GTCCACCACCCTGTTGCT GTAG-3'). RT-PCR assays were performed on a QuantStudio ${ }^{\mathrm{TM}} 7$ Flex Real-Time PCR system (Applied Biosystems) using SYBR Green MasterMix (ABI). For accurate normalization of quantitative data, quantification was carried out using GAPDH mRNA as an internal standard.

\section{Cell Culture and Treatment}

The human renal tubular epithelial cells (HK-2) were purchased from the American Type Culture Collection (Wuhan Academy of Life Sciences, Wuhan, China). The cells were maintained in DMEM consisting of $10 \%$ fetal bovine serum, $100 \mathrm{U} / \mathrm{mL}$ penicillin, and $100 \mathrm{U} / \mathrm{mL}$ streptomycin. Cells were grown in a humidified atmosphere at $37^{\circ} \mathrm{C}$ with $5 \% \mathrm{CO}_{2}$ and $95 \% \mathrm{O}_{2}$. After 3-5 passages, the cells were trypsin-dissociated and seeded onto collagen-coated 96 -well plates at a density of $0.5-1 \times 10^{5}$. The cell viability was measured using Cell Counting Kit-8 assay and normalized as the percentage of control.

\section{Measurement of Intracellular ROS Level}

The total intracellular ROS were determined by the $2^{\prime}, 7^{\prime}$. dichlorodihydrofluorescein diacetate (H2DCFDA) assay. In brief, HK-2 cells were seeded in a 96-well plate and followed by pretreatment with AS-IV $(25,50$ and $100 \mu \mathrm{M}$ ) (Wang et al., 2019a; Wang et al., 2019b) for $30 \mathrm{~min}$, and then co-treatment with tacrolimus $(15 \mu \mathrm{M})$ at $37^{\circ} \mathrm{C}$ for $24 \mathrm{~h}$. The cells were loaded with H2DCFDA. The fluorescence images were taken using a fluorescence microscope (System Microscopy IX70; Olympus, Tokyo, Japan) $30 \mathrm{~min}$ after. Fluorescent signals intensities of cells were counted using Image-Pro Plus (IPP) software.

\section{Small Interfering (siRNA) Transfection}

For silencing the p62 proteins, HK-2 cells were transfected with $10 \mathrm{pmol}$ of siRNAs for p62 (Invitrogen) using Lipofectamine RNAiMAX (Invitrogen) following the manufacturer's instructions. And unconjugated control siRNA (Invitrogen) was used for control experiments. The detailed experimental protocols were provided in supplementary materials.

\section{Statistical Analysis}

All data are presented as mean \pm SD. The results were statistically evaluated using two tailed Student's t test, one-way analysis of variance (ANOVA) followed by least significant difference (LSD) post hoc test. Statistical significance was set at $p<0.05$.

\section{RESULTS}

\section{Effects of AS-IV on Tacrolimus-Induced Mice Renal Dysfunction}

To evaluate the effects of AS-IV on TIN, mice were given AS-IV at the dose of 10,20 and $40 \mathrm{mg} / \mathrm{kg}$, respectively. Although treatment with tacrolimus for 28 days did not impact animal weight gain or the urine volume, it remarkably increased the levels of SCr and BUN $(p<0.01)$ (Table 1). Whereas, AS-IV at 20 or $40 \mathrm{mg} / \mathrm{kg} / \mathrm{d}$ restored the levels of SCr and BUN $(p<0.01)$, and no significant difference was found between the two groups. ASIV at $10 \mathrm{mg} / \mathrm{kg} / \mathrm{d}$ failed to attenuate tacrolimus-induced renal dysfunction.

\section{Effects of AS-IV on Tacrolimus-Induced Mice Tubulointerstitial Fibrosis}

$\mathrm{HE}$ and Masson trichrome staining of paraffin-embedded kidney tissue indicated that neither inflammation nor tubulointerstitial fibrosis was observed in the control group (Figure 1A). However, treatment with tacrolimus resulted in vacuolar and granular degeneration of tubular epithelial cells, tubular atrophy, tubular cast formation, extensive inflammatory cell infiltration and tubulointerstitial fibrosis. AS-IV at $10 \mathrm{mg} / \mathrm{kg} / \mathrm{d}$ mildly improved the above-mentioned pathological changes of renal tubules and interstitium, and AS-IV at 20 and $40 \mathrm{mg} / \mathrm{kg} / \mathrm{d}$ obviously ameliorated it. Further quantitative analysis of 
TABLE 1 | Effect of Astragaloside IV on tacrolimus-induced chronic nephrotoxicity in mice $(n=8)$.

\begin{tabular}{|c|c|c|c|c|}
\hline & Body weight (g) & Urine volume $(\mathrm{ml} / \mathrm{d})$ & $\operatorname{Scr}(\mu \mathrm{mol} / L)$ & BUN (mmol/L) \\
\hline Control & $26.5 \pm 0.6$ & $0.92 \pm 0.09$ & $37.5 \pm 5.9$ & $8.5 \pm 1.0$ \\
\hline Tac & $24.5 \pm 1.0$ & $1.09 \pm 0.17$ & $64.9 \pm 11.9^{\star \star}$ & $14.5 \pm 3.8^{\star \star}$ \\
\hline Tac + AS-IV 10 & $25.1 \pm 0.8$ & $0.98 \pm 0.15$ & $55.4 \pm 12.2$ & $12.0 \pm 3.9$ \\
\hline Tac +AS-IV 20 & $27.1 \pm 0.7$ & $1.01 \pm 0.10$ & $44.2 \pm 6.1^{\# \#}$ & $9.0 \pm 1.5^{\# \#}$ \\
\hline Tac + AS-IV 40 & $26.8 \pm 0.6$ & $1.02 \pm 0.15$ & $41.1 \pm 7.3^{\# \#}$ & $8.7 \pm 1.8^{\# \#}$ \\
\hline
\end{tabular}

Abbreviations: Scr, serum creatinine; BUN, blood urea nitrogen; Tac, tacrolimus; AS-IV, Astragaloside IV

The values are presented as means $\pm S D$. ${ }^{* *} \mathrm{p}<0.01$ vs. the Control group; ${ }^{\# \#} \mathrm{p}<0.01$ vs. the Tac group.

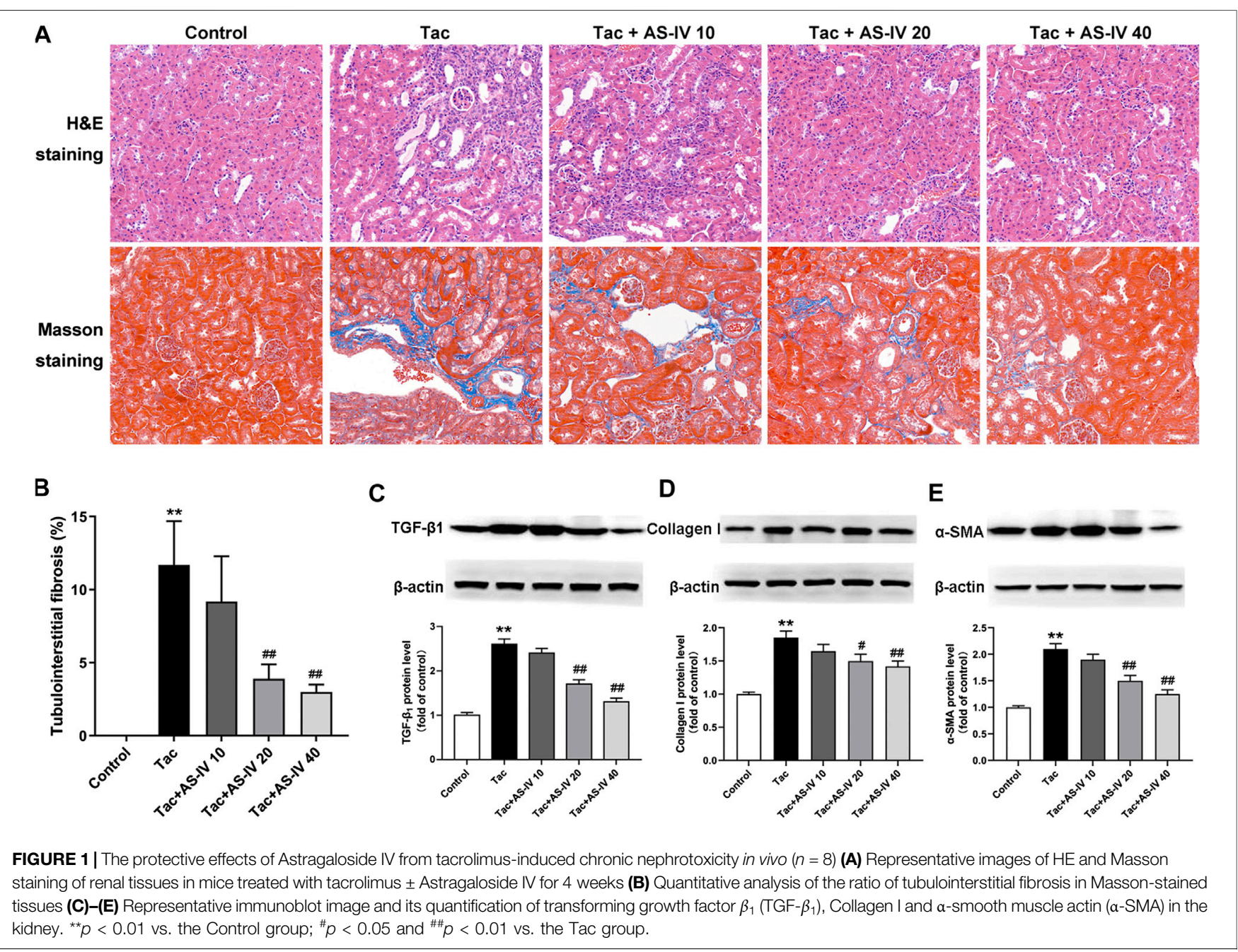

Masson trichrome-stained tissues suggested that AS-IV (20 and $40 \mathrm{mg} / \mathrm{kg} / \mathrm{d}$ ) significantly reduced the proportion of tacrolimusinduced tubulointerstitial fibrosis $(p<0.01)$ (Figure 1B). Notably, the renal-protection activity of AS-IV $20 \mathrm{mg} / \mathrm{kg} / \mathrm{d}$ was not significantly different from that of $40 \mathrm{mg} / \mathrm{kg} / \mathrm{d}(p>0.05)$. In addition, the biomarkers for TIN were quantified to verify the effects of AS-IV. As shown in Figures 1C-E, tacrolimus significantly increased the expression of TGF- $\beta_{1}$, collagen I and $\alpha$-SMA in the kidney compared to the control group $(p<$
0.01). In line with the pathological findings, AS-IV co-treatment $(20$ and $40 \mathrm{mg} / \mathrm{kg} / \mathrm{d}$ ) remarkably prevented the upregulation of these biomarkers $(p<0.05)$.

\section{AS-IV Attenuated Tacrolimus-Induced Oxidative Stress in Mice}

Tacrolimus caused a significant increased level of MDA as compared to the control group $(p<0.01)$, which was 

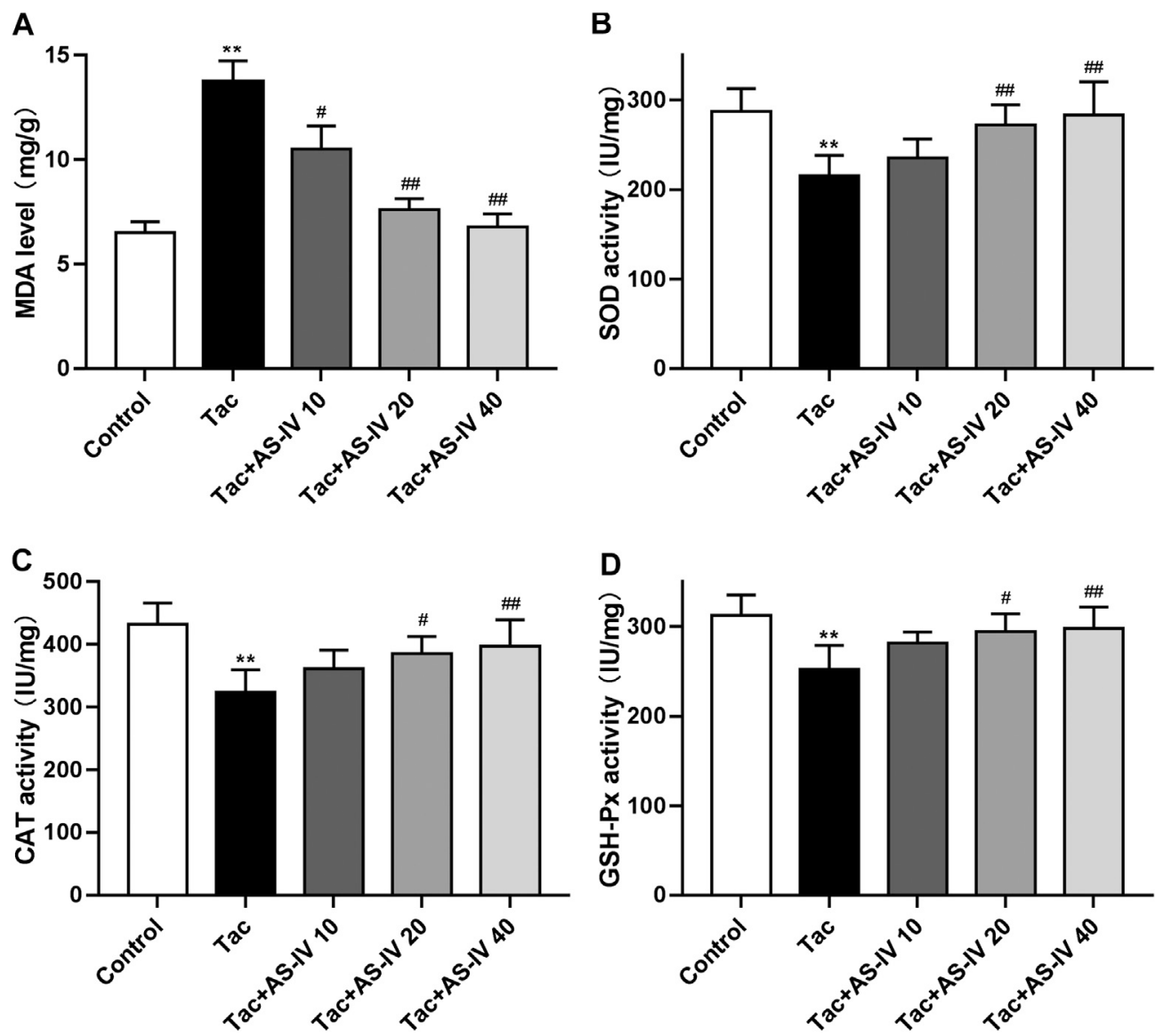

FIGURE 2|Astragaloside IV attenuates oxidative stress induced by tacrolimus in vivo $(n=8)$. Mice were treated with tacrolimus \pm Astragaloside IV for 4 weeks, and then renal tissues were taken to evaluate the effects of Astragaloside IV on malondialdehyde (MDA) (A), superoxidase dismutase (SOD) (B), catalase (CAT) (C) and glutathione peroxidase (GSH-Px) (D) levels. ${ }^{* *} p<0.01$ vs. the Control group; ${ }^{*} p<0.05$ and ${ }^{\# \#} p<0.01$ vs. the Tac group.

remarkably reduced by the treatment of AS-IV at all three doses (Figure 2A). Besides, tacrolimus administration caused prominent decrease in activity of antioxidant enzymes (SOD, CAT and GSH-Px) in kindey tissue, which was remarkably enhanced by AS-IV treatment at 20 and $40 \mathrm{mg} / \mathrm{kg} / \mathrm{d}$ (Figures 2B-D). The above in vivo data indicated that AS-IV could significantly improve TIN at a dose of $20 \mathrm{mg} / \mathrm{kg}$, so this dose was used for subsequent mechanism studies.

\section{AS-IV Reduced Tacrolimus-Induced Cell Death and Oxidative Stress in HK-2 Cells}

To confirm the protective effect of AS-IV, we evaluated the impact of AS-IV on TIN in HK-2 cells. First, the AS-IV (25, 50 and $100 \mu \mathrm{M}$ ) did not decrease the cell viability (Figure 3A). Compared with the control group, the viability of HK-2 cells treated with $15 \mu \mathrm{M}$ tacrolimus for $24 \mathrm{~h}$ was decreased to $67.3 \pm$ $4.5 \%$ (Figure 3B). Whereas, AS-IV at the concentrations of 50 and $100 \mu \mathrm{M}$ protected cells against tacrolimus-induced injury in a dose-dependent manner. The potential involvement of ROS in the cytoprotective effect of AS-IV against tacrolimus-induced renal injury was subsequently examined. As shown in Figures 3C,D, tacrolimus increased the intracellular ROS levels by 1.67 fold compared with the control group, while AS-IV, especially at 50 and $100 \mu \mathrm{M}$, significantly reduced the up-regulation of ROS levels caused by tacrolimus $(p<0.01)$.

\section{AS-IV Protected TIN via Increasing Nuclear Nrf2 Accumulation in vivo}

Since Nrf2 is crucial in regulating the transcription of plenty of antioxidant genes, we next examined whether the Nrf2 pathway was activated by AS-IV. RT-PCR assays showed that neither tacrolimus nor AS-IV significantly affected the mRNA levels of Nrf2 $(p>0.05)$ (Figure 4A). Tacrolimus hardly affected the protein levels of $\mathrm{Nrf} 2(p>0.05)$, while AS-IV significantly raised it $(p<0.05)$ (Figures 4B,C). Moreover, the mRNA expression of HO-1, NQO1 and GCLC, the downstream targets of Nrf2, were significantly upregulated by AS-IV treatment (Figures 4D-F). Considering that Nrf2 activates its target gene expression as a transcriptional factor only in the nucleus, we therefore determine the nuclear translocation of $\mathrm{Nrf} 2$ in renal tissue. As shown in 

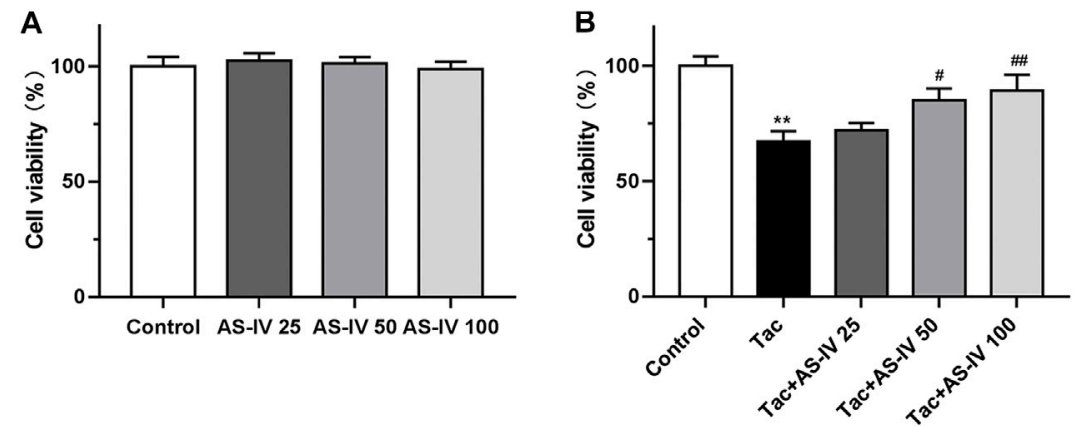
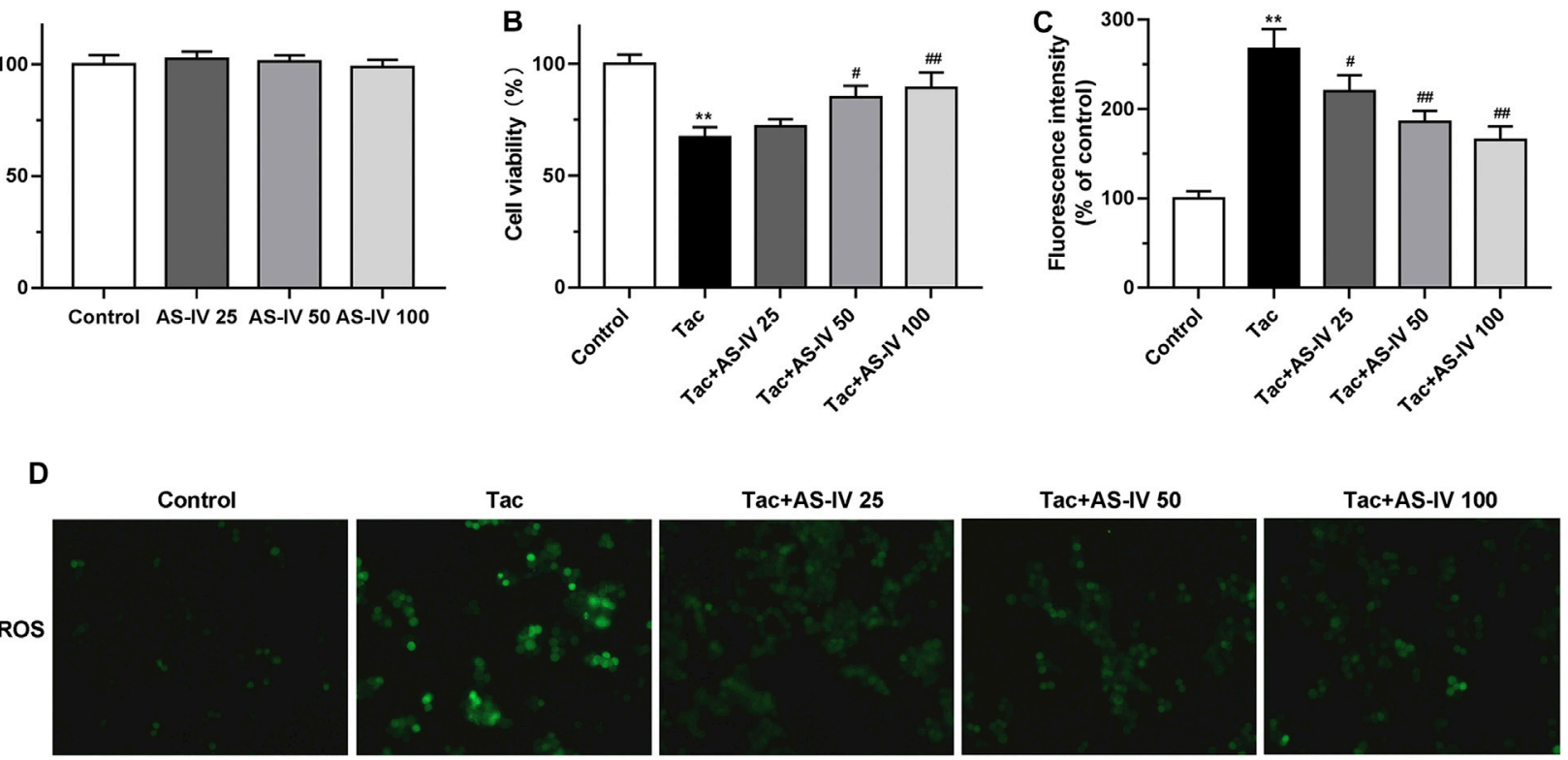

FIGURE 3 The cell protective and ROS scavenge effect of Astragaloside IV in HK-2 cells (A-B) HK-2 cells were treated with Astragaloside IV (25, 50 or 100 $\mu$ M) \pm tacrolimus $(15 \mu \mathrm{M})$ for $24 \mathrm{~h}$, and then the cell viability was measured using Cell Counting Kit- 8 assay. The results were calculated from three independent experiments (C-D) The levels of intracellular ROS were detected with $2^{\prime}, 7^{\prime}$-dichlorodihydrofluorescein diacetate (H2DCFDA) assay. Results were calculated by the intensity of eight fields from three independent experiments. ${ }^{* \star} p<0.01$ vs. the Control group; ${ }^{\#} p<0.05$ and ${ }^{\# \#} p<0.01$ vs. the Tac group.
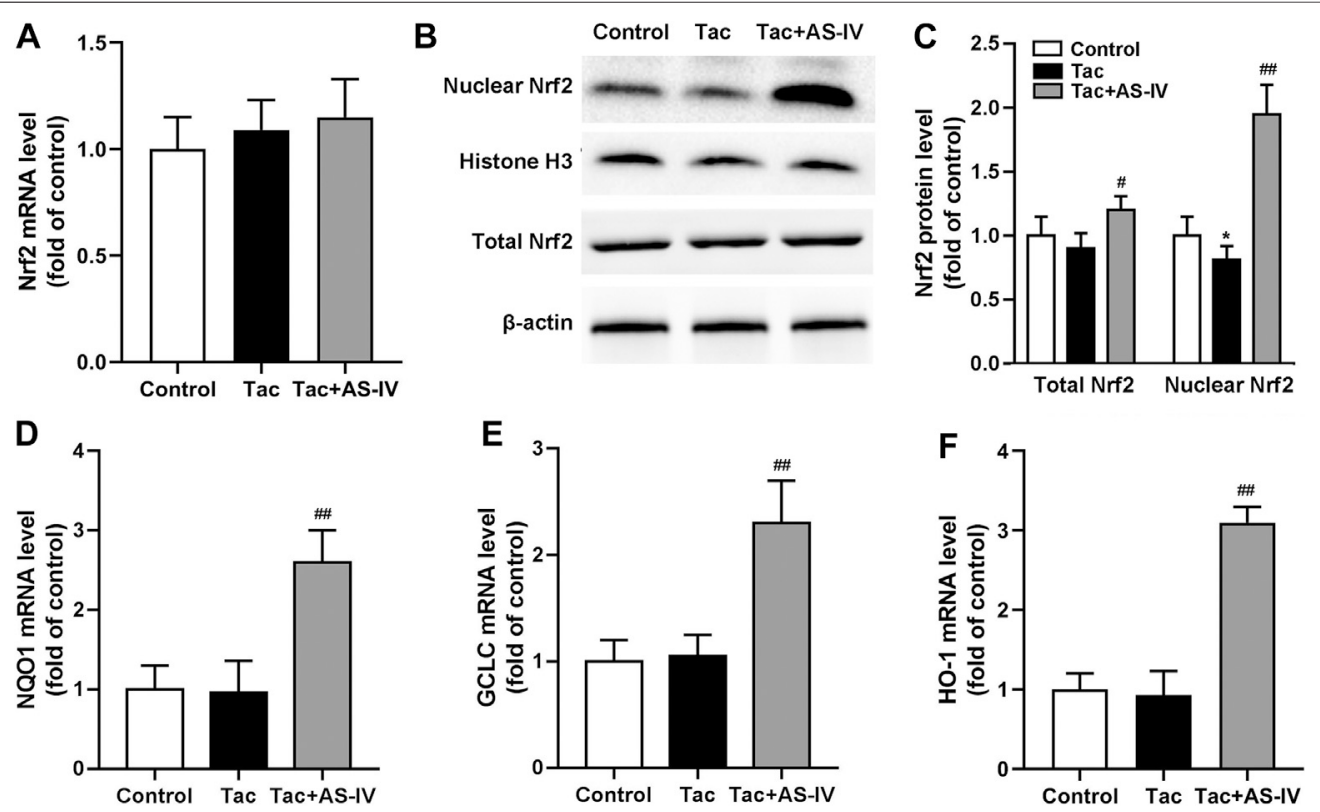

FIGURE 4 | Astragaloside IV induced Nrf2 nuclear translocation and down-stream genes transcription in vivo. The mice were treated with tacrolimus \pm Astragaloside IV for 4 weeks, and then renal tissues were taken to evaluate the effects of Astragaloside IV on the Nrf2 mRNA levels ( $n=8$ ) (A), the nuclear and total Nrf2 protein levels $(n=6)(\mathbf{B})-(\mathbf{C})$, the mRNA levels of NQO1 (D), GCLC (E) and HO-1 (F) $(n=8) .{ }^{*} p<0.05$ vs. the Control group; ${ }^{\#} p<0.05$ and ${ }^{\# \#} p<0.01$ vs. the Tac group.

Figures $4 \mathrm{~B}, \mathrm{C}$, the protein levels of $\mathrm{Nrf} 2$ in the nucleus was decreased by treatment with tacrolimus $(p<0.05)$, but dramatically elevated by the co-administration of AS-IV $(p<$
0.01). These results indicate that AS-IV abrogates tacrolimusinduced oxidative stress injury may by promoting Nrf2 transfer into the nucleus. 

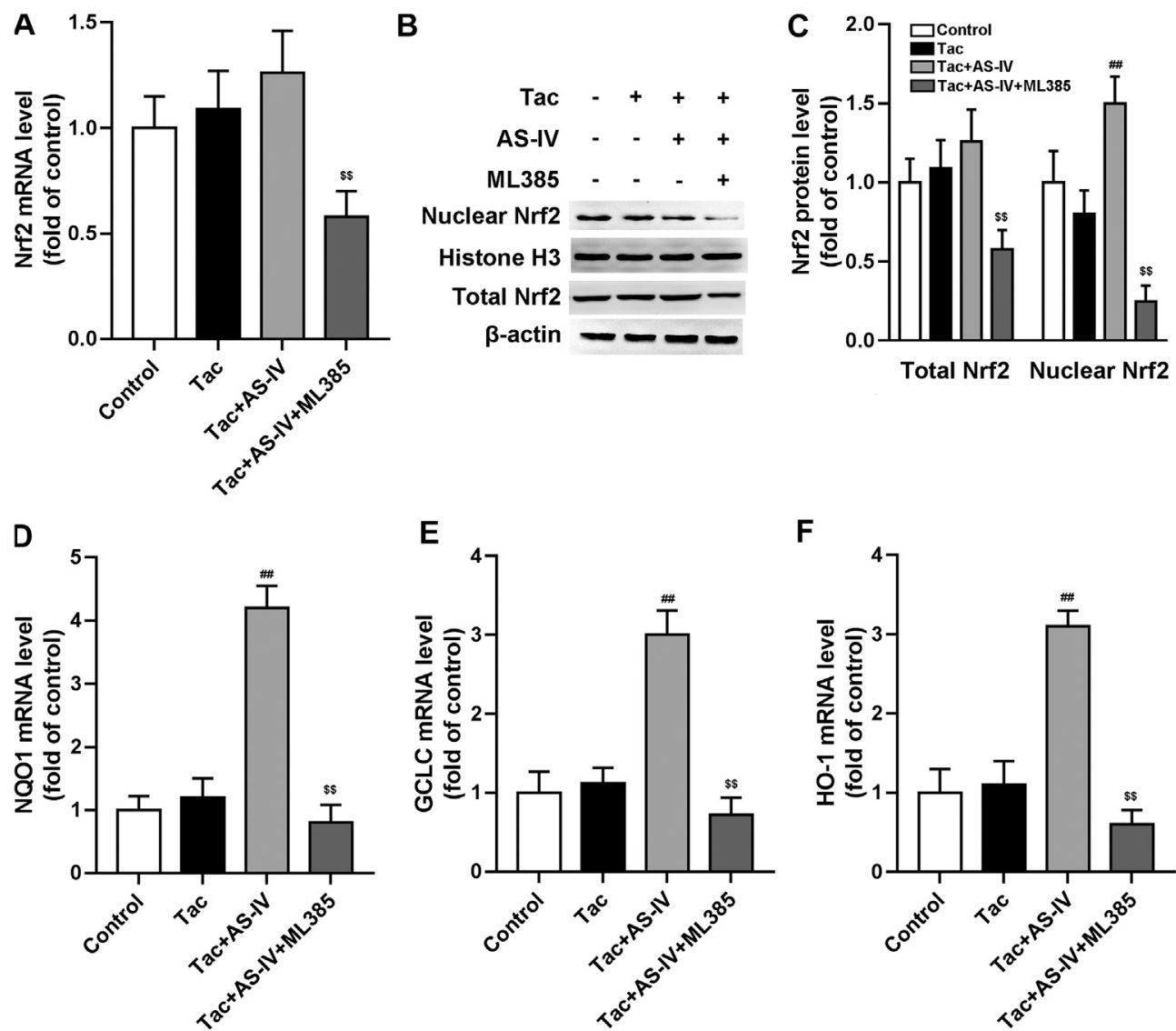

FIGURE 5 | ML385 inhibited Astragaloside IV-induced Nrf2 nuclear translocation and down-stream genes transcription in HK-2 cells. The HK-2 cell were pretreated with AS-IV $(50 \mu \mathrm{M})$ with or without ML385 $(3 \mu \mathrm{M})$ for $30 \mathrm{~min}$. Then tacrolimus was added into the cells for $24 \mathrm{~h}$ (A) The Nrf2 mRNA in HK-2 cells were tested by RT-qPCR assay $(n=8)$ (B-C) The nuclear Nrf2 and total Nrf2 protein of HK-2 cell were detected by Western blotting ( $n=6)$. The mRNA levels of NQO1 (D), GCLC (E) and HO-1 (F) were evaluated by RT-qPCR $(n=8) .{ }^{\# \#} p<0.01$ vs. the Tac group; ${ }^{\$} p<0.01$ vs. the Tac + AS-IV group.

\section{Nrf2 Was Required for AS-IV to Protect HK-2 Cells From TIN}

To verify whether Nrf2 was dominantly involved in the antioxidative stress activity of AS-IV, HK-2 cells were treated with or without Nrf2 inhibitor ML385. Consistent with the in vivo data, AS-IV $(50 \mu \mathrm{M})$ markedly induced nuclear Nrf2 accumulation instead of increasing its mRNA levels in HK-2 cells (Figures 5A-C). The downstream antioxidant genes of Nrf2, such as $\mathrm{HO}-$ 1, NQO1, and GCLC, were also increased by AS-IV (Figures 5D-F). Whereas, these effects were significantly abrogated by ML385 in the HK-2 cells (Figures 5A-F). Hence, Nrf2 played a critical role in the prevention against TIN by AS-IV.

\section{AS-IV Increased p62 Phosphorylation and Its Interaction With Keap1, Leading to Nrf2 Activation}

AS-IV increased the protein level of Nrf2 without impacting its mRNA, which indicating that AS-IV may function by reducing the degradation of Nrf2. Since Keap1 is the most critical protein that regulates Nrf2 degradation (Cuadrado et al., 2019), the expression of Keap1 was examined. As shown in Figure 6A, mRNA levels of Keap1 were unchanged in both tacrolimus and AS-IV-treated renal tissues. However, the protein levels of Keap1 were obviously decreased after AS-IV treatment compared with that in the model group $(p<0.01)$ (Figures 6B,C).

p62 is well characterized for its ability to recruit and sequester Keap1 to autophagosomes for degradation (Lee et al., 2020). Therefore, the levels of p62 in kidney were subsequently determined. The mRNA levels of p62 were marginally affected by tacrolimus $(p>0.05)$, but significantly induced by AS-IV $(p<$ 0.05) (Figure 6D). Unexpectedly, the protein levels of p62 were increased by tacrolimus $(p<0.01)$, but hardly affected by AS-IV (Figures 6E,F). Therefore, we sought an additional mechanism for the decreased Keap1 by AS-IV. Binding affinity between Keap 1 and p62 is known to be modulated by phosphorylation of p62 at a specific amino-acid residue, serine 351 (S351) in mice (S349 in humans) (Ichimura et al., 2013; Deng et al., 2020; Lee et al., 2020). Given that, AS-IV may mediate S351 phosphorylation of p62. Thus, we measured the levels of p62 phosphorylation. As shown in Figures 6E,F, tacrolimus didn't affect the levels of p62 phosphorylation, while AS-IV dramatically increased the phosphorylation of $\mathrm{p} 62(p<0.01)$. These results 

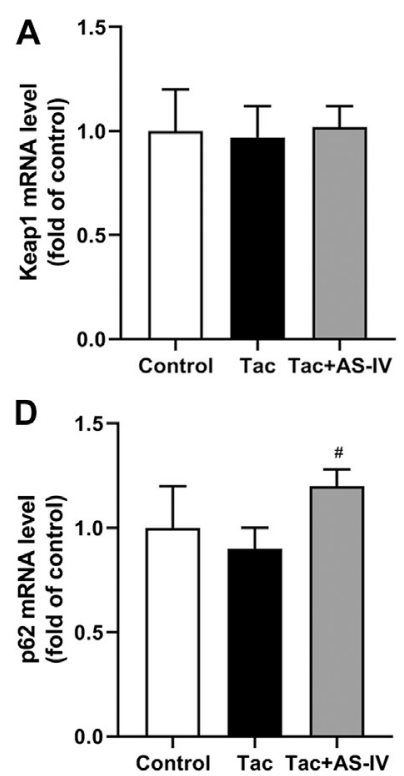

B

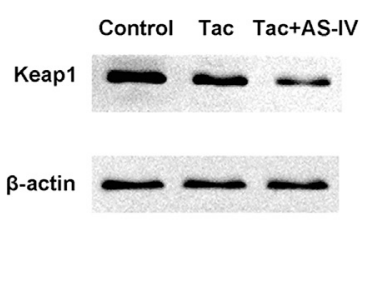

E

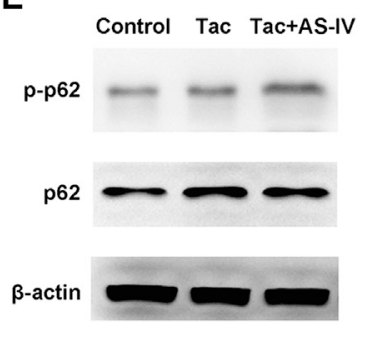

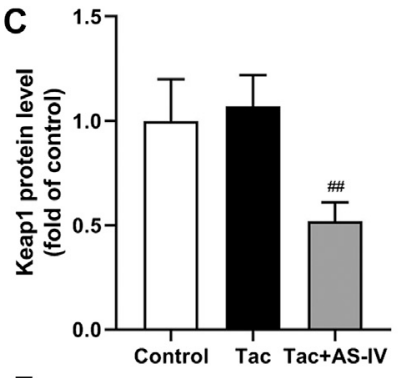

$\mathbf{F}$

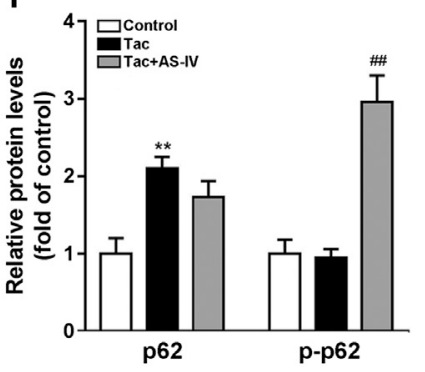

FIGURE 6 | Astragaloside IV increased Keap1 degradation and p62 phosphorylation in vivo. Mice were treated with tacrolimus \pm Astragaloside IV for 4 weeks, and then renal tissues were taken to evaluate the effects of Astragaloside IV on the Keap1 mRNA levels $(n=8)(\mathbf{A})$, the Keap1 protein levels $(n=6) \mathbf{( B )}$-(C), the p62 mRNA levels $(n=8)$ (D) and the p62 as well as phosphorylated p62 protein levels $(n=6)(\mathbf{E}-\mathbf{F}) .{ }^{* *} p<0.01$ vs. the Control group; ${ }^{\#}<<0.05$ and ${ }^{\# \#} p<0.01$ vs. the Tac group.

indicated that AS-IV increased the binding between Keap1 and p62 by facilitating p62 phosphorylation.

\section{Genetic Knockdown of p62 Abrogated the Activity of AS-IV on Nrf2 Nuclear Translocation}

To verify the role of p62, HK-2 cells were transfected with p62 siRNA. After p62 was abrogated, AS-IV failed to decrease the protein level of Keap1, and accordingly Nrf2 nuclear accumulation was cancelled (Figures 7A-C). p62 siRNA also weakened the AS-IV induced up-regulation of antioxidant genes including HO-1, NQO1, and GCLC (Figures 7D-F). Taken together, these gain- and loss-of-function data suggest that p62 is essential for AS-IV-mediated protective effects against oxidative stress-related TIN.

\section{DISCUSSION}

The chronic tacrolimus exposure is associated with an increased risk of $\mathrm{CKD}$, which hinders its long-term use in transplant recipients (Ojo et al., 2003). Despite years of research, no specific treatment for TIN is available. In the present study, we found AS-IV significantly attenuated TIN via minimizing tacrolimus-induced oxidative stress. The anti-oxidative property of AS-IV was causally associated with the activation of p62Keap1-Nrf2 pathway. These findings suggested that AS-IV was a promising prophylactic or therapeutic option for TIN.

As one of the main active components of Astragalus, AS-IV possesses various pharmacological activities, such as neuroprotection, liver and kidney protection (Zhang et al.,
2020). Although its oral bioavailability is relatively low $(7.4 \%$ in beagle dogs and $3.7 \%$ in rats) and is mainly eliminated by liver $(0.0041 / \mathrm{kg} / \mathrm{min})$, AS-IV owns the highest concentration in liver and kidney (Zhang et al., 2006; Chang et al., 2012). At present, a few studies have explored its role in drug-associated acute nephrotoxicity (Yan et al., 2017; Qu et al., 2020). Nevertheless, the pathogenesis of acute and chronic nephrotoxicity is known to vary considerably (Mehta et al., 2015), so its role in drug-induced chronic nephrotoxicity remains unknown. In this study, AS-IV at 20 and $40 \mathrm{mg} / \mathrm{kg} / \mathrm{d}$ remarkably reduced the ratio of tubulointerstitial fibrosis $(p<0.01)$ which is a well-recognized marker for TIN (Yu et al., 2019). In addition, tacrolimus-induced increase in SCr $(p<0.01)$, BUN $(p<0.01)$ and fibrosis-related proteins $(p<0.05)$, including TGF- $\beta_{1}$, Collagen I and $\alpha$-SMA, were significantly restored by AS-IV. Unfortunately, there has not been a well-recognized effective positive drug used in the researches on TIN until now. Therefore, like most studies (Lee et al., 2018; Lim et al., 2019b; Yu et al., 2019), these markers can only be compared between the treatment group and model group. Despite this limitation, these results can at least indicate that ASIV was effective for TIN.

AS-IV has been proved to act as a reactive oxygen species (ROS) scavenger to relieve kidney injury in diabetic nephropathy (Du et al., 2018; Wang et al., 2020). Meanwhile, oxidative stress is believed to play a critical role in TIN (Lim et al., 2017; Yu et al., 2019). Hence, we investigated the role of oxidative stress in the renal protection of AS-IV. Our findings indicated that tacrolimus significantly increased the level of MDA $(p<0.01)$ which was a product of phospholipids peroxidation and caused oxidative stress in cells (Lim et al., 2015). And tacrolimus markedly inhibited the activities of antioxidant stress enzymes such as SOD, CAT and GSH-Px $(p<0.01)$. Whereas the MDA level and 

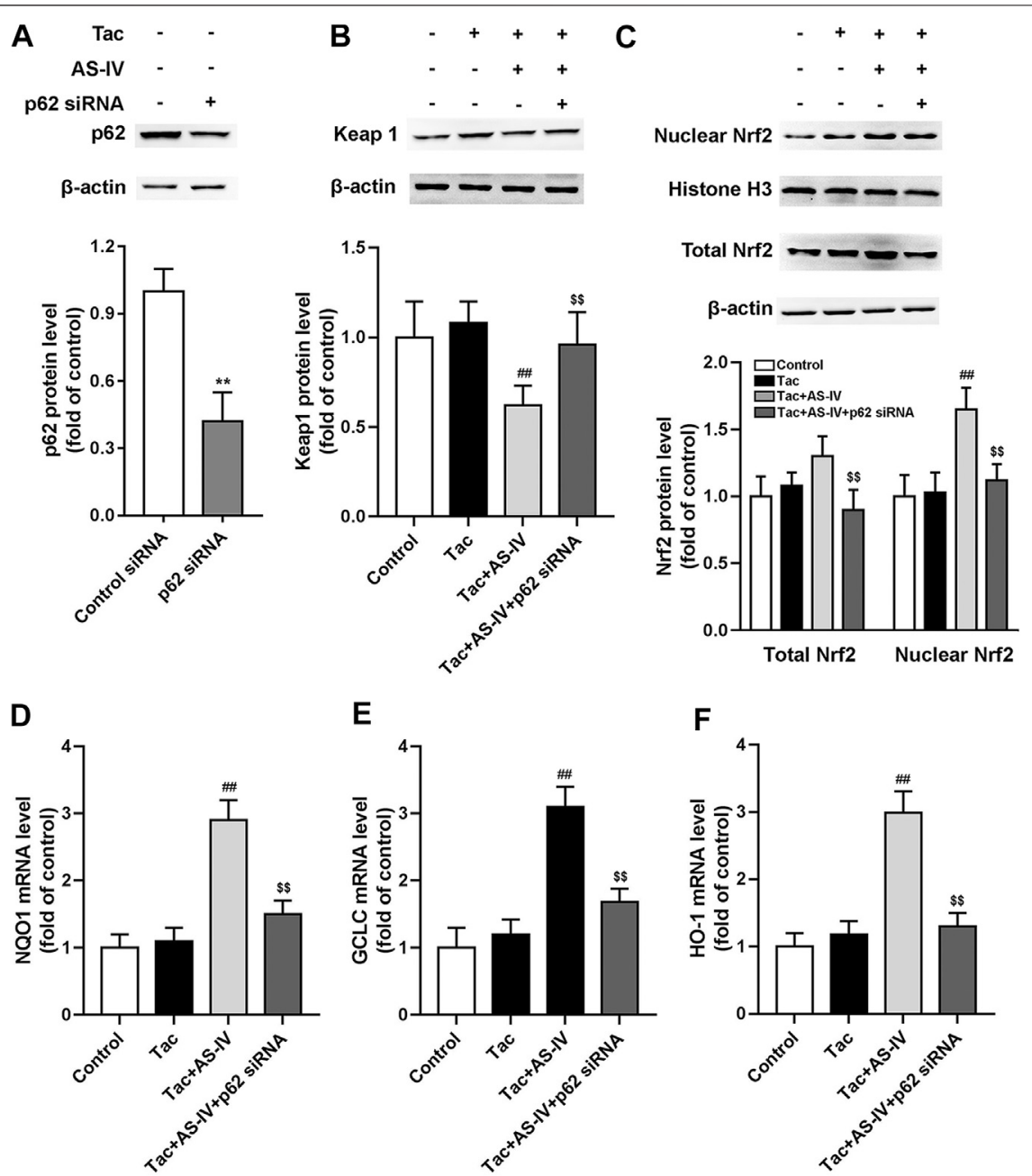

FIGURE 7 | p62 siRNA inhibited Astragaloside IV-induced Nrf2 nuclear translocation and down-stream genes transcription in HK-2 cells via p62-Keap1-Nrf2 pathway. The HK-2 cells were transfected with unconjugated control siRNA or p62 siRNA, and then the protein levels of p62 $(n=4)$ (A), Keap1 $(n=6)$ (B), and the nuclear Nrf2 as well as total Nrf2 $(n=6)$ (C) were detected. The mRNA levels of NQO1 (D), GCLC (E) and HO-1 (F), were evaluated by RT-qPCR $(n=8)$. ${ }^{* \star} p<0.01$ vs. the Control group; ${ }^{\# \# p} 0.01$ vs. the Tac group; ${ }^{\$ \$} p<0.01$ vs. the Tac + AS-IV group.

the antioxidant enzyme activity were obviously restored by AS-IV at 20 and $40 \mathrm{mg} / \mathrm{kg} / \mathrm{d}(p<0.05)$. The results in HK-2 cells confirmed that tacrolimus significantly increased intercellular ROS levels $(p<0.01)$, while AS-IV significantly reduced it $(p<0.05)$. The in vivo and in vitro studies suggested that the protective effect of AS-IV against TIN may be associated with antioxidant stress.

It is well documented that the transcriptional activation of antioxidant proteins is dominantly regulated by the redoxsensitive transcription factor Nrf2. Although antioxidant stress has been proved to be effective in ameliorating TIN (Lim et al., 2017; Lim et al., 2019a; Luo et al., 2019), the role of Nrf2 in TIN has rarely been researched. However, we failed to observed that tacrolimus impacted the expression of Nrf2 $(p>0.05)$. Since Nrf2 remains inactive in the cytoplasm under basal conditions and only functions after translocating into the nucleus (Cuadrado et al., 2019), we tested the distribution of Nrf2 in the nucleus. And then we found tacrolimus significantly decreased the protein levels of Nrf2 in the nucleus $(p<0.05)$. AS-IV significantly induced Nrf2 nuclear translocation $(p<0.01)$ and its downstream target genes such as HO-1, NQO1 and GCLC $(p<0.01)$ both in kidney tissues and in HK-2 cells. To further investigate the causal relationship between Nrf2 and AS-IV's protection against TIN, ML385, an identified chemical compound that specifically binds to the Neh1 domain of Nrf2 and inhibits its downstream target gene expression (Singh et al., 2016), was used. After the pretreatment of ML385, the promotion of AS-IV on Nrf2 nuclear translocation $(p<0.01)$ and its target genes transcription $(p<0.01)$ were abolished. These results confirmed that Nrf2 activation is the key to relieving TIN by AS-IV.

AS-IV increased the protein level of Nrf2 instead of its mRNA level, implying that AS-IV may function by weakening the degradation of Nrf2. It is well-established that $\mathrm{Nrf} 2$ is degraded by the Keap1-Cul3 E3 ubiquitin ligase complex through polyubiquitination (Cuadrado et al., 2019). Therefore, 
we determined the expression of Keap1, and then found that ASIV remarkably reduced its protein level both in vivo and in vitro $(p<0.01)$. p62, a critical autophagy-adaptor protein, has a Keap1interacting region (KIR) domain, which allows p62 to sequester Keap1 into the autophagosomes and promote its degradation via autophagy (Lee et al., 2020). However, the binding affinity of nonphosphorylated p62 for Keap1 was two orders of magnitude weaker than that of Nrf2. Phosphorylation of p62 at Ser351 could enhance p62 affinity for Keap1 binding to a comparable level to that of Nrf2 (Ichimura et al., 2013; Deng et al., 2020). Hence, we detected the levels of p62 and phosphorylated p62, and found that AS-IV markedly raised the latter $(p<0.01)$. Subsequently, siRNAmediated silencing of $\mathrm{p} 62$ was performed to verify the role of $\mathrm{p} 62$. In HK-2 cells, p62 siRNA knockdown resulted in a sharply decline of AS-IV-induced Nrf2 nuclear accumulation, which indicated that AS-IV activated Nrf2 in a p62 dependent manner. Interestingly, tacrolimus led to an increase of p62 protein levels, whereas AS-IV marginally decreased its expression. This result cannot be explained by the positive feedback p62-Keap1-Nrf2 loop in which Nrf2 facilitates p62 expression (Lee et al., 2020). Previous research has found tacrolimus could inhibit autophagic flow and resulted in accumulation of p62 (Lim et al., 2019b). Accordingly, we speculate that AS-IV may reduce p62 content by activating autophagic flow, which needs to be confirmed by further research in the future.

In summary, the present study confirms the protective effects of AS-IV against TIN in mice for the first time. This renal protective activity is at least partially attributable to AS-IVmediated induction of $\mathrm{p} 62$ phosphorylation, thereby increasing its competition with Nrf2 for Keap1 binding, and then facilitating Nrf2 nucleus translocation, alleviating ROS accumulation and

\section{REFERENCES}

Cao, Y., Zhang, L., Wang, Y., Fan, Q., and Cong, Y. (2019). Astragaloside IV attenuates renal fibrosis through repressing epithelial-to-mesenchymal transition by inhibiting microRNA-192 expression: in vivo and in vitro studies. Am. J. Transl. Res. 11 (8), 5029-5038. doi:10.1002/path.2961

Chang, Y. X., Sun, Y. G., Li, J., Zhang, Q. H., Guo, X. R., Zhang, B. L., et al. (2012). The experimental study of Astragalus membranaceus on meridian tropsim: the distribution study of astragaloside IV in rat tissues. J. Chromatogr. B Analyt. Technol. Biomed. Life Sci. 911, 71-75. doi:10. 1016/j.jchromb.2012.10.024

Chapman, J. R. (2011). Chronic calcineurin inhibitor nephrotoxicity-lest we forget. Am. J. Transpl. 11 (4), 693-697. doi:10.1111/j.1600-6143.2011.03504.x

Chen, Q., Su, Y., Ju, Y., Ma, K., Li, W., and Li, W. (2018). Astragalosides IV protected the renal tubular epithelial cells from free fatty acids-induced injury by reducing oxidative stress and apoptosis. Biomed. Pharmacother, 108, 679-686. doi:10.1016/j.biopha.2018.09.049

Cuadrado, A., Rojo, A. I., Wells, G., Hayes, J. D., Cousin, S. P., Rumsey, W. L., et al. (2019). Therapeutic targeting of the NRF2 and KEAP1 partnership in chronic diseases. Nat. Rev. Drug Discov. 18 (4), 295-317. doi:10.1038/s41573-0180008-x

Deng, Z., Lim, J., Wang, Q., Purtell, K., Wu, S., Palomo, G. M., et al. (2020). ALSFTLD-linked mutations of SQSTM1/p62 disrupt selective autophagy and NFE2L2/NRF2 anti-oxidative stress pathway. Autophagy 16 (5), 917-931. doi:10.1080/15548627.2019.1644076

Du, N., Xu, Z., Gao, M., Liu, P., Sun, B., and Cao, X. (2018). Combination of Ginsenoside Rg1 and Astragaloside IV reduces oxidative stress and inhibits renal fibrosis. Given the superior therapeutic efficacy of AS-IV in TIN, AS-IV may be developed as a promising candidate drug for the prevention and treatment of TIN.

\section{DATA AVAILABILITY STATEMENT}

The raw data supporting the conclusions of this article will be made available by the authors, without undue reservation.

\section{ETHICS STATEMENT}

The animal study was reviewed and approved by Puai Hospital Animal Care and Use Committee.

\section{AUTHOR CONTRIBUTIONS}

PG, CZ, and XG contributed to the study conception and design. PG, XD, LL, and XG performed the experiment and acquired the primary data. HX, ML, and CZ helped to interpret the data. PG and XD drafted the manuscript. All authors contributed to the article and approved the submitted version.

\section{FUNDING}

This work was supported by grants from the Wuhan Health and Family Planning Commission (No. WZ18Q03 to PG) and National Natural Scientific Foundation of China (NSFC, No. 81803503 to $\mathrm{XG}$ ).

TGF-beta1/Smads signaling cascade on renal fibrosis in rats with diabetic nephropathy. Drug Des. Dev. Ther. 12, 3517-3524. doi:10.2147/DDDT.S171286

Gao, P., Guan, X. L., Huang, R., Shang-Guan, X. F., Luan, J. W., Liu, M. C., et al. (2020). Risk factors and clinical characteristics of tacrolimus-induced acute nephrotoxicity in children with nephrotic syndrome: a retrospective casecontrol study. Eur. J. Clin. Pharmacol. 76 (2), 277-284. doi:10.1007/s00228019-02781-3

Guo, H., Cao, A., Chu, S., Wang, Y., Zang, Y., Mao, X., et al. (2016). Astragaloside IV attenuates podocyte apoptosis mediated by endoplasmic reticulum stress through upregulating sarco/endoplasmic reticulum $\mathrm{Ca}(2+)$-ATPase 2 expression in diabetic nephropathy. Front. Pharmacol. 7, 500. doi:10.3389/ fphar.2016.00500

Hart, A., Smith, J. M., Skeans, M. A., Gustafson, S. K., Wilk, A. R., Castro, S., et al. (2019). OPTN/SRTR 2017 annual data report: kidney. Am. J. Transplant 19 (Suppl. 1), 219-123. doi:10.1111/ajt.15274

Ichimura, Y., Waguri, S., Sou, Y. S., Kageyama, S., Hasegawa, J., Ishimura, R., et al. (2013). Phosphorylation of p62 activates the Keap1-Nrf2 pathway during selective autophagy. Mol. Cell 51 (5), 618-631. doi:10.1016/j.molcel.2013.08.003

Lee, D. H., Park, J. S., Lee, Y. S., Han, J., Lee, D.-K., Kwon, S. W., et al. (2020) SQSTM1/p62 activates NFE2L2/NRF2 via ULK1-mediated autophagic KEAP1 degradation and protects mouse liver from lipotoxicity. Autophagy, 108, 1-25. doi:10.1080/15548627.2020.1712108

Lee, D., Lee, D. S., Jung, K., Hwang, G. S., Lee, H. L., Yamabe, N., et al. (2018) Protective effect of ginsenoside $\mathrm{Rb} 1$ against tacrolimus-induced apoptosis in renal proximal tubular LLC-PK1 cells. J. Ginseng Res. 42 (1), 75-80. doi:10. 1016/j.jgr.2016.12.013

Lim, S. W., Jin, L., Luo, K., Jin, J., Shin, Y. J., Hong, S. Y., et al. (2017). Klotho enhances FoxO3-mediated manganese superoxide dismutase expression by 
negatively regulating PI3K/AKT pathway during tacrolimus-induced oxidative stress. Cell Death Dis. 8 (8), e2972. doi:10.1038/cddis.2017.365

Lim, S. W., Jin, L., Piao, S. G., Chung, B. H., and Yang, C. W. (2015). Inhibition of dipeptidyl peptidase IV protects tacrolimus-induced kidney injury. Lab. Invest. 95 (10), 1174-1185. doi:10.1038/labinvest.2015.93

Lim, S. W., Shin, Y. J., Luo, K., Quan, Y., Cui, S., Ko, E. J., et al. (2019a). Ginseng increases Klotho expression by FoxO3-mediated manganese superoxide dismutase in a mouse model of tacrolimus-induced renal injury. Aging ( $N$. Y.) 11 (15), 5548-5569. doi:10.18632/aging.102137

Lim, S. W., Shin, Y. J., Luo, K., Quan, Y., Ko, E. J., Chung, B. H., et al. (2019b). Effect of Klotho on autophagy clearance in tacrolimus-induced renal injury. FASEB. J. 33 (2), 2694-2706. doi:10.1096/fj.201800751R

Lin, S., An, X., Guo, Y., Gu, J., Xie, T., Wu, Q., et al. (2019). Meta-analysis of astragalus-containing traditional Chinese medicine combined with chemotherapy for colorectal cancer: efficacy and safety to tumor response. Front Oncol, 13 (9), 749. doi:10.3389/fonc.2019.00749

Luo, K., Lim, S. W., Jin, J., Jin, L., Gil, H. W., Im, D. S., et al. (2019). Cilastatin protects against tacrolimus-induced nephrotoxicity via anti-oxidative and antiapoptotic properties. BMC Nephrol 20 (1), 221. doi:10.1186/s12882-019-1399-6

Lusco, M. A., Fogo, A. B., Najafian, B., and Alpers, C. E. (2017). AJKD atlas of renal pathology: calcineurin inhibitor nephrotoxicity. Am. J. Kidney Dis. 69 (5), e21-e22. doi:10.1053/j.ajkd.2017.02.003

Mehta, R. L., Awdishu, L., Davenport, A., Murray, P. T., Macedo, E., Cerda, J., et al. (2015). Phenotype standardization for drug-induced kidney disease. Kidney Int. 88 (2), 226-234. doi:10.1038/ki.2015.115

Naesens, M., Kuypers, D. R., and Sarwal, M. (2009). Calcineurin inhibitor nephrotoxicity. Clin. J. Am. Soc. Nephrol. 4 (2), 481-508. doi:10.2215/CJN. 04800908

Ojo, A. O., Held, P. J., Port, F. K., Wolfe, R. A., Leichtman, A. B., Young, E. W., et al. (2003). Chronic renal failure after transplantation of a nonrenal organ. N. Engl. J. Med. 349 (10), 931-940. doi:10.1056/NEJMoa021744

Qu, X., Gao, H., Zhai, J., Sun, J., Tao, L., Zhang, Y., et al. (2020). Astragaloside IV enhances cisplatin chemosensitivity in hepatocellular carcinoma by suppressing MRP2. Eur. J. Pharmaceut. Sci. 148, 105325. doi:10.1016/j. ejps.2020.105325

Sawinski, D., Trofe-Clark, J., Leas, B., Uhl, S., Tuteja, S., Kaczmarek, J. L., et al. (2016). Calcineurin inhibitor minimization, conversion, withdrawal, and avoidance strategies in renal transplantation: a systematic review and metaanalysis. Am. J. Transplant 16 (7), 2117-2138. doi:10.1111/ajt.13710

Singh, A., Venkannagari, S., Oh, K. H., Zhang, Y. Q., Rohde, J. M., Liu, L., et al. (2016). Small molecule inhibitor of NRF2 selectively intervenes therapeutic resistance in KEAP1-deficient NSCLC tumors. ACS Chem. Biol. 11 (11), 3214-3225. doi:10.1021/acschembio.6b00651

Wang, E., Wang, L., Ding, R., Zhai, M., Ge, R., Zhou, P., et al. (2020). Astragaloside IV acts through multi-scale mechanisms to effectively reduce diabetic nephropathy. Pharmacol. Res. 157, 104831. doi:10.1016/j.phrs.2020.104831
Wang, X., Gao, Y., Tian, N., Wang, T., Shi, Y., Xu, J., et al. (2019a). Astragaloside IV inhibits glucose-induced epithelial-mesenchymal transition of podocytes through autophagy enhancement via the SIRT-NF-kB p65 axis. Sci. Rep. 9 (1), 323. doi:10.1038/s41598-018-36911-1

Wang, Y., Jiang, F., Cheng, H., Tan, X., Liu, Y., Wei, C., et al. (2019b). Astragaloside IV protects against oxidative stress in calf small intestine epithelial cells via NFE2L2-antioxidant response element signaling. Int. J. Mol. Sci. 20 (24), 6131. doi:10.3390/ijms20246131

Yan, W., Xu, Y., Yuan, Y., Tian, L., Wang, Q., Xie, Y., et al. (2017). Renoprotective mechanisms of Astragaloside IV in cisplatin-induced acute kidney injury. Free Radic. Res. 51 (7-8), 669-683. doi:10.1080/10715762.2017.1361532

Yu, J. H., Lim, S. W., Luo, K., Cui, S., Quan, Y., Shin, Y. J., et al. (2019). Coenzyme Q10 alleviates tacrolimus-induced mitochondrial dysfunction in kidney. FASEB. J 33 (11), 12288-12298. doi:10.1096/fj.201900386RR

Zhang, D. D., and Chapman, E. (2020). The role of natural products in revealing NRF2 function. Nat. Prod. Rep. 37, 797-826, doi:10.1039/C9NP00061E

Zhang, J., Wu, C., Gao, L., Du, G., and Qin, X. (2020). Astragaloside IV derived from Astragalus membranaceus: a research review on the pharmacological effects. Adv. Pharmacol 87, 89-112. doi:10.1016/bs.apha.2019.08.002

Zhang, L., Shergis, J. L., Yang, L., Zhang, A. L., Guo, X., Zhang, L., et al. (2019). Astragalus membranaceus (Huang Qi) as adjunctive therapy for diabetic kidney disease: an updated systematic review and meta-analysis. J. Ethnopharmacol 239. 111921. doi:10.1016/j.jep.2019.111921

Zhang, S., Liu, Y., Xiang, D., Yang, J., Liu, D., Ren, X., et al. (2018). Assessment of dose-response relationship of 5-fluorouracil to murine intestinal injury. Biomed. Pharmacother 106, 910-916. doi:10.1016/j.biopha.2018.07.029

Zhang, W. D., Zhang, C., Liu, R. H., Li, H. L., Zhang, J. T., Mao, C., et al. (2006). Preclinical pharmacokinetics and tissue distribution of a natural cardioprotective agent astragaloside IV in rats and dogs. Life Sci. 79 (8), 808-815. doi:10.1016/j.lfs.2006.02.032

Zhou, X., Sun, X., Gong, X., Yang, Y., Chen, C., Shan, G., et al. (2017). Astragaloside IV from Astragalus membranaceus ameliorates renal interstitial fibrosis by inhibiting inflammation via TLR4/NF- $\mathrm{KB}$ in vivo and in vitro. Int. Immunopharm 42, 18-24. doi:10.1016/j.intimp.2016.11.006

Conflict of Interest: The authors declare that the research was conducted in the absence of any commercial or financial relationships that could be construed as a potential conflict of interest.

Copyright $\odot 2021 \mathrm{Gao}, \mathrm{Du}, \mathrm{Liu}, \mathrm{Xu}, \mathrm{Liu}, \mathrm{Guan}$ and Zhang. This is an open-access article distributed under the terms of the Creative Commons Attribution License (CC $B Y)$. The use, distribution or reproduction in other forums is permitted, provided the original author(s) and the copyright owner(s) are credited and that the original publication in this journal is cited, in accordance with accepted academic practice. No use, distribution or reproduction is permitted which does not comply with these terms. 